\title{
Modulating Noncovalent and Covalent Forces to Control Inverse Phosphocholine Lipid Self-Assembly on Inorganic Surfaces
}

Tun Naw Sut ${ }^{\dagger, \ddagger}$, Abdul Rahim Ferhan ${ }^{\dagger}$, Soohyun Park ${ }^{\dagger}$, Dong Jun Koo ${ }^{\ddagger}$, Bo Kyeong Yoon', Joshua A. Jackman*,+, Nam-Joon Cho*,ं

${ }^{\dagger}$ School of Materials Science and Engineering, Nanyang Technological University, 50 Nanyang Drive 637553, Singapore

${ }^{\ddagger}$ School of Chemical Engineering and Translational Nanobioscience Research Center, Sungkyunkwan University, Suwon 16419, Republic of Korea

School of Healthcare and Biomedical Engineering, Chonnam National University, Yeosu, 59626, Republic of Korea

E-mail: njcho@ntu.edu.sg and jjackman@skku.edu

\begin{abstract}
$\underline{\text { Abstract }}$
While noncovalent forces typically drive lipid vesicle adsorption and rupture to form supported lipid bilayer (SLB) coatings on inorganic surfaces, this strategy only works on a few materials with suitable energetics such as $\mathrm{SiO}_{2}$. The use of coordination chemistry between inversephosphocholine (PC) lipid headgroups and surfaces has emerged as a promising strategy to enable SLB formation on other materials such as $\mathrm{TiO}_{2}$ based on covalent forces. However, until now, a cohesive picture of how noncovalent and covalent forces jointly contribute to the latter SLB formation process has been lacking. Herein, we investigated inverse-PC lipid vesicle adsorption onto $\mathrm{TiO}_{2}$ and $\mathrm{SiO}_{2}$ surfaces and discovered how adsorption pathways can be controlled by tuning the balance of noncovalent and covalent forces. On $\mathrm{TiO}_{2}$, SLB formation depended on two key factors: (1) favorable noncovalent forces to facilitate initial vesicle adsorption; and (2) a critical density of lipid- $\mathrm{TiO}_{2}$ coordinate bonds to enable sufficient vesicle deformation triggering fusion and rupture. In other cases, either no adsorption or intact vesicle adsorption without rupture occurred even when coordinate bonds were present. Conversely, on $\mathrm{SiO}_{2}$, conditions were identified to support inverse-PC lipid adsorption whereas vesicles were repelled otherwise. The experimental results were supported by interfacial force modeling and our findings demonstrate how a subtle interplay of noncovalent and covalent forces plays a deterministic role in modulating lipid self-assembly pathways.
\end{abstract}

Keywords: vesicle; adsorption; supported lipid bilayer; phospholipid; noncovalent; coordinate bonding; interfacial forces 


\section{Introduction}

Understanding how phospholipid membranes interact with inorganic materials is critical to various applications such as biosensors and drug delivery tools, and can enable the design of functional biointerfaces such as ultrathin supported lipid bilayer (SLB) coatings. ${ }^{1-3}$ SLBs are usually composed of naturally occurring, biocompatible phosphatidylcholine (PC) lipids as the main component and can impart antifouling properties as well as aid selective biofunctionalization. ${ }^{4,5}$ Such efforts fit within the nanoarchitectonics concept to control the molecular self-assembly of phospholipids with a defined nanoscale organization at a solid-liquid interface. ${ }^{6,7}$ The most common approach to SLB fabrication involves the noncovalent adsorption and spontaneous rupture of nanoscopic lipid vesicles at solid-liquid interfaces. ${ }^{8-12}$ Interestingly, this self-assembly process can only occur on a narrow range of surfaces with favorable material properties and mechanistic details continue to be unraveled through experimental, simulation, and theoretical approaches. ${ }^{13-15}$ There is extensive interest in deciphering why lipid vesicles adsorb and rupture on certain hydrophilic surfaces, but not others, and in translating such chemical insights into the design of improved SLB biointerfaces with tailored properties and stabilities.

The prevailing notion is that zwitterionic PC lipid vesicles can adsorb and rupture spontaneously on $\mathrm{SiO}_{2}$ surfaces to form an SLB coating while they typically adsorb and remain intact on $\mathrm{TiO}_{2}$ surfaces. ${ }^{16}$ The distinct self-assembly outcomes are believed to arise from differences in vesiclesurface interaction strength. ${ }^{17-20} \mathrm{On} \mathrm{SiO}_{2}$, adsorbed PC lipid vesicles undergo more extensive deformation due to stronger vesicle-surface interactions, which makes them more prone to fusion and rupture. ${ }^{20}$ Conversely, adsorbed vesicles have weaker interactions with $\mathrm{TiO}_{2}$ surfaces, resulting in less deformation. ${ }^{21}$ The different extents of vesicle deformation have been measured experimentally $^{22}$ (see also related work on SLB systems ${ }^{23}$ ) and the surface-dependent variation in vesicle-surface interactions has also been discussed in the context of noncovalent interfacial forces, including van der Waals, double-layer electrostatic, and steric-hydration forces. ${ }^{24}$ To fabricate SLBs on $\mathrm{TiO}_{2}$ surfaces, there have been efforts to enhance vesicle-surface interactions by virtue of changing solution $\mathrm{pH}^{25}$ along with utilizing alternative approaches that bypass the need for deformation-mediated vesicle rupture. ${ }^{26,27}$

The aforementioned efforts have relied on noncovalent strategies that result in weakly attached SLBs while there have also been creative strategies to fabricate SLB coatings on $\mathrm{TiO}_{2}$ surfaces by utilizing coordination chemistry - a line of research that not only has practical utility but also sheds light on the fundamental chemistry of phospholipid membranes on inorganic surfaces. ${ }^{28}$ These strategies have been centered around inverse-PC lipids, which are structurally similar to PC lipids but have a flipped headgroup whereby the quaternary amine is connected to the glycerol backbone and the anionic phosphate group is presented outward. ${ }^{29}$ The main example of an inverse-PC lipid is 2-((2,3-bis(oleoyloxy)propyl)dimethylammonio)ethyl hydrogen phosphate (DOCP) and negatively charged DOCP lipid vesicles have been reported to fuse with $\mathrm{TiO}_{2}$ nanoparticles to form SLB coatings. ${ }^{30,31}$

Strikingly, DOCP lipid vesicles do not fuse with $\mathrm{SiO}_{2}$ nanoparticles, which is an opposite trend to that observed with zwitterionic PC lipid vesicles that fuse with $\mathrm{SiO}_{2}$, but not $\mathrm{TiO}_{2}$, nanoparticles. ${ }^{30}$ The distinct interaction behavior of DOCP lipid vesicles has been attributed to coordinate covalent bond formation between phosphate groups and titanol (Ti-O) groups, which enhances vesiclesurface interactions on $\mathrm{TiO}_{2}$ surfaces, whereas DOCP lipid vesicles are generally repelled from $\mathrm{SiO}_{2}$ surfaces. ${ }^{32}$ It has also been shown that DOCP lipid vesicles can fuse with $\mathrm{TiO}_{2}$ nanoparticles 
across a wide range of $\mathrm{pH}$ conditions, reinforcing the importance of coordinate bond formation while the inability of DOCP lipid vesicles to fuse with $\mathrm{SiO}_{2}$ nanoparticles has been attributed to charge repulsion. ${ }^{32}$ As such, existing efforts have viewed covalent and noncovalent forces as discrete factors in driving DOCP lipid vesicle interactions with $\mathrm{TiO}_{2}$ and $\mathrm{SiO}_{2}$ surfaces, respectively. However, this mechanistic picture is likely incomplete, especially in the $\mathrm{TiO}_{2}$ case, as it is known that noncovalent forces play a universally important role in vesicle-surface adhesion processes. Hence, there is an outstanding need to unify the mechanistic picture of how noncovalent interfacial forces and coordination chemistry contribute to DOCP lipid vesicle interactions with inorganic surfaces.

Towards this goal, herein, we scrutinized the real-time adsorption kinetics of $\sim 75$-nm diameter, DOCP-containing lipid vesicles onto flat, macroscopic $\mathrm{TiO}_{2}$ and $\mathrm{SiO}_{2}$ surfaces and unraveled how the interplay of noncovalent and covalent forces in this system plays a deterministic role in modulating lipid self-assembly pathways. While coordination chemistry has been viewed as a critical factor in enabling DOCP-containing lipid vesicles to fuse with and form SLBs on $\mathrm{TiO}_{2}$ surfaces, our findings reveal a more nuanced situation whereby noncovalent forces mediate initial adsorption and a minimum density of coordinate bonds between vesicles and the surface must form to enable a sufficiently high degree of vesicle deformation that leads to vesicle fusion and rupture.

\section{$\underline{\text { Results }}$}

\section{Measurement Strategy}

We utilized the quartz crystal microbalance-dissipation (QCM-D) technique to characterize the nanoscale mass and viscoelastic properties of lipid adlayers that formed on the $\mathrm{TiO}_{2}-$ and $\mathrm{SiO}_{2}-$ coated sensor surfaces and the corresponding adsorption kinetics could be temporally tracked based on the simultaneously recorded resonance frequency $(\Delta \mathrm{f})$ and energy dissipation $(\Delta \mathrm{D})$ measurement signals, respectively. ${ }^{33}$ This label-free characterization method allowed us to directly probe DOCP lipid interactions with inorganic surfaces in real-time, complementing past nanoparticle studies ${ }^{30-32}$ that mainly focused on measuring fluorescent dye release from within vesicles and dye attached to phospholipids that indirectly inferred vesicle fusion behavior and lipid attachment, respectively.

\section{Ionic Strength Effects}

Using the QCM-D technique, we first measured 100 mol\% DOCP lipid vesicle adsorption onto $\mathrm{TiO}_{2}$ and $\mathrm{SiO}_{2}$ surfaces in $10 \mathrm{mM}$ Tris buffer [pH 7.5] with different $\mathrm{NaCl}$ concentrations, which mainly influenced the degree of electrostatic interactions based on charge screening and hence modulated the overall degree of noncovalent forces in the system. On $\mathrm{TiO}_{2}$ surfaces, the $\Delta \mathrm{f}$ and $\triangle \mathrm{D}$ signals indicated that vesicles adsorbed in $100 \mathrm{mM}$ and higher $\mathrm{NaCl}$ concentrations with twostep kinetics, whereas nearly negligible adsorption occurred in $50 \mathrm{mM} \mathrm{NaCl}$ (Figure 1A). The corresponding final $\Delta \mathrm{f}$ and $\Delta \mathrm{D}$ shifts of the lipid adlayers formed in 100 to $250 \mathrm{mM} \mathrm{NaCl}$ conditions were around -24 to $-26 \mathrm{~Hz}$ and less than $0.3 \times 10^{-6}$, respectively, which indicate SLB formation $^{8}$ (Figure 1B). Together, these QCM-D measurement responses are consistent with vesicle adsorption and spontaneous rupture on the $\mathrm{TiO}_{2}$ surface.

In marked contrast, the final $\Delta \mathrm{f}$ and $\Delta \mathrm{D}$ shifts of the lipid adlayer formed in the $50 \mathrm{mM} \mathrm{NaCl}$ condition were only around $-6 \mathrm{~Hz}$ and less than $0.3 \times 10^{-6}$, respectively, which point to nearly negligible adsorption. Hence, DOCP lipid vesicle adsorption onto $\mathrm{TiO}_{2}$ surfaces led to SLB 

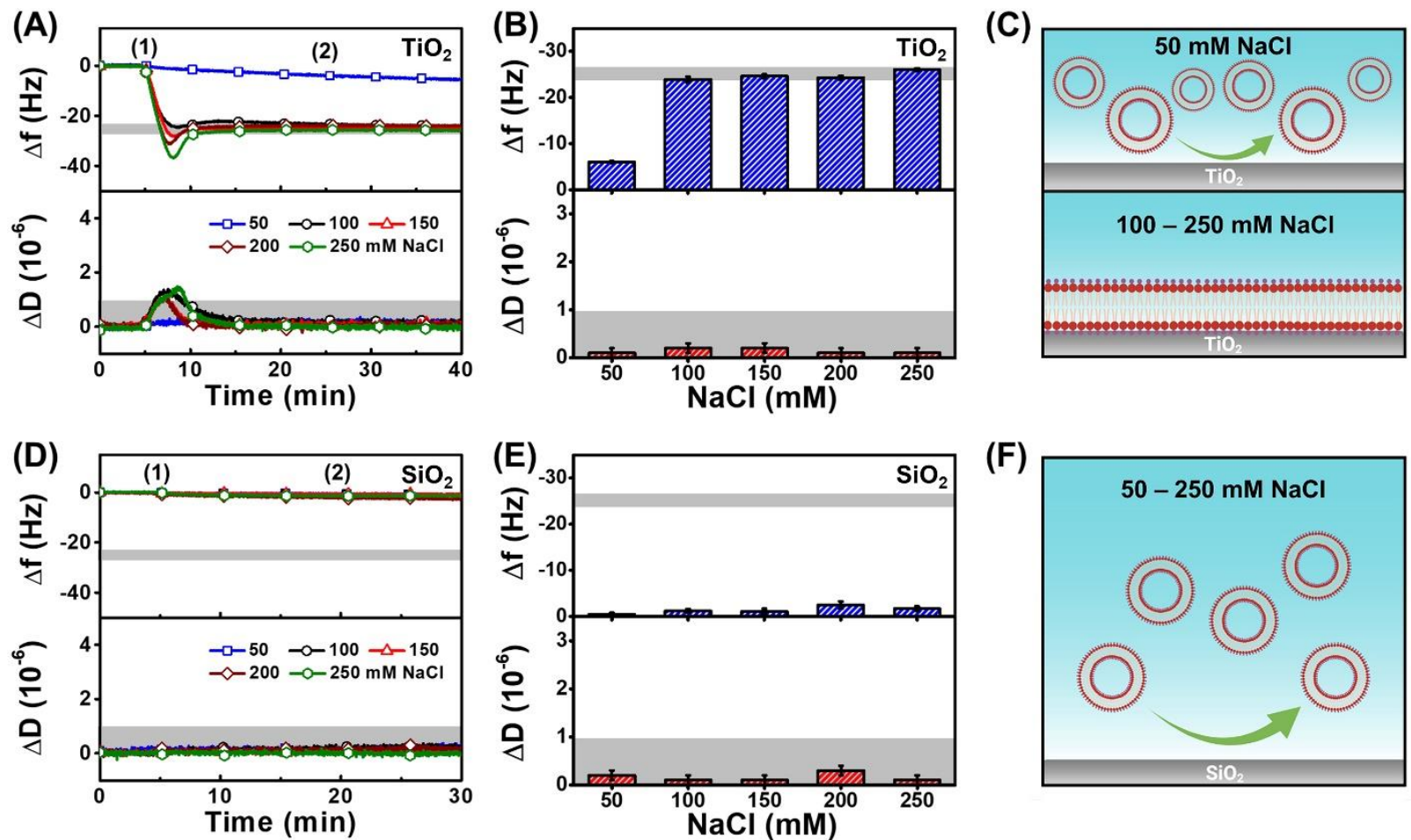

Figure 1. Effect of $\mathrm{NaCl}$ concentration on DOCP lipid vesicle adsorption onto $\mathrm{TiO}_{2}$ and $\mathrm{SiO}_{2}$ surfaces. (A) QCM-D frequency $(\Delta \mathrm{f})$ and energy dissipation $(\Delta \mathrm{D})$ signals upon DOCP lipid vesicle addition to $\mathrm{TiO}_{2}$ surface. Labels (1) and (2) refer to vesicle addition and buffer washing, respectively. (B) Summary of final $\Delta \mathrm{f}$ and $\Delta \mathrm{D}$ shifts for adsorbed lipid layers on $\mathrm{TiO}_{2}$ surface. Mean and standard deviation are reported from at least three measurements. Shaded regions in the graphs indicate typical measurement values for an SLB. (C) Illustration of vesicle adsorption outcomes on $\mathrm{TiO}_{2}$ surface. (D-F) Corresponding data and illustration for DOCP lipid vesicle addition to $\mathrm{SiO}_{2}$ surface.

formation in $100-250 \mathrm{mM} \mathrm{NaCl}$ conditions and there was minimal adsorption in the $50 \mathrm{mM} \mathrm{NaCl}$ condition (Figure 1C). While the phosphate moieties of the DOCP lipid headgroups are known to readily form coordinate covalent bonds with the $\mathrm{TiO}_{2}$ surface, ${ }^{32}$ the observed dependence on $\mathrm{NaCl}$ concentration supports that noncovalent vesicle-surface interactions play a critical role in controlling the initial adsorption process. Since the DOCP lipid vesicles and $\mathrm{TiO}_{2}$ surface are both negatively charged at $\mathrm{pH} \mathrm{7.5,},^{25,} 29$ these findings further support that salt-mediated charge screening modulates the degree of electrostatic repulsion between contacting vesicles and the surface.

On the other hand, on $\mathrm{SiO}_{2}$ surfaces, there was negligible DOCP lipid vesicle adsorption at all tested $\mathrm{NaCl}$ conditions (Figure 1D). The corresponding final $\Delta \mathrm{f}$ and $\Delta \mathrm{D}$ shifts were less than -4 $\mathrm{Hz}$ and $0.3 \times 10^{-6}$, respectively, which indicate minimal adsorption (Figure 1E). Since the $\mathrm{SiO}_{2}$ surface is also negatively charged at $\mathrm{pH} 7.5,{ }^{25}$ this finding supports that there is a relatively larger degree of electrostatic repulsion between DOCP lipid vesicles and the $\mathrm{SiO}_{2}$ surface. The lack of vesicle adsorption on the $\mathrm{SiO}_{2}$ surface also agrees well with a past study that showed DOCP lipid vesicles had low adsorption onto $\mathrm{SiO}_{2}$ nanoparticles in the $0-100 \mathrm{mM} \mathrm{NaCl}$ concentration range. ${ }^{30}$ Hence, the results support that DOCP lipid vesicles do not adsorb onto the $\mathrm{SiO}_{2}$ surface across the tested $\mathrm{NaCl}$ concentration range (Figure 1F). 
To complement the QCM-D experiments, we performed analytical calculations to estimate how the noncovalent interfacial forces that underpin DOCP lipid vesicle-surface attachment are influenced by $\mathrm{NaCl}$ concentration trendwise. The calculations modeled the vesicle-surface contact region as a planar lipid bilayer on top of a flat surface and considered the van der Waals, doublelayer electrostatic, and steric-hydration forces according to extended-DLVO (E-DLVO) theory. ${ }^{34,}$ 35 The vesicle-surface interaction energy was plotted as a function of the separation distance between the vesicle and surface in order to identify the minimum interaction energy, which corresponded to an equilibrium separation distance. ${ }^{35} \mathrm{In}$ the $\mathrm{TiO}_{2}$ case, the plots showed that there were attractive interactions between DOCP lipid vesicles and the $\mathrm{TiO}_{2}$ surface for $100 \mathrm{mM}$ and higher $\mathrm{NaCl}$ concentrations, as indicated by stable energy minima at around $2 \mathrm{~nm}$ separation distance (Figure 2A). On the other hand, the calculations showed that there was only a weak interaction between the DOCP lipid vesicles and the $\mathrm{TiO}_{2}$ surface in the $50 \mathrm{mM} \mathrm{NaCl}$ case, which agrees well with the experimental result indicating negligible adsorption ( $c f$. Figure 1). Trendwise, the minimum interaction energy became more favorable and shifted from around -20 to $-36 \mu \mathrm{J} / \mathrm{m}^{2}$ as the $\mathrm{NaCl}$ concentration increased from 100 to $250 \mathrm{mM}$, whereas the minimum interaction energy was almost negligible at around $-4 \mu \mathrm{J} / \mathrm{m}^{2}$ in the $50 \mathrm{mM} \mathrm{NaCl}$ case (Figure 2B).
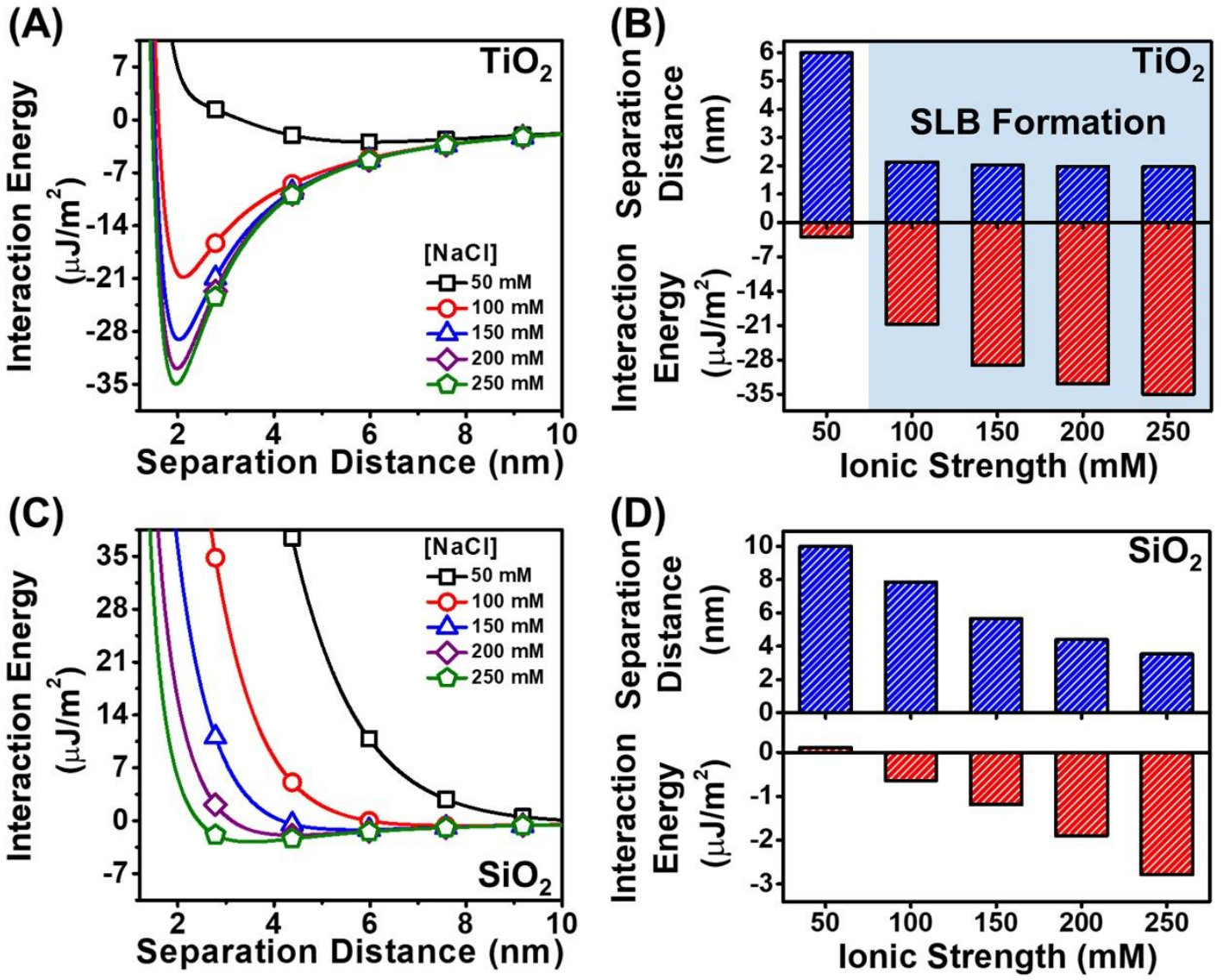

Figure 2. Extended-DLVO model analysis of DOCP lipid vesicle interactions with $\mathrm{TiO}_{2}$ and $\mathrm{SiO}_{2}$ surfaces. (A) Dependence of the total interaction energy on the vesicle-surface separation distance for different $\mathrm{NaCl}$ concentrations. (B) Summary values of equilibrium separation distance and interaction energy for adsorbed vesicles on a $\mathrm{TiO}_{2}$ surface. Shaded region corresponds to $\mathrm{NaCl}$ concentrations at which a DOCP SLB formed. (C-D) Corresponding data for adsorbed vesicles on an $\mathrm{SiO}_{2}$ surface. 
In addition, in the $\mathrm{SiO}_{2}$ case, the plots indicated that there were negligible interactions between DOCP lipid vesicles and the $\mathrm{SiO}_{2}$ surface across the tested range of $\mathrm{NaCl}$ concentrations, which is consistent with the experimental results (Figure 2C). The interaction energy tended to marginally increase at higher $\mathrm{NaCl}$ concentrations due to charge screening, however, the minimum interaction energy was always smaller than $-4 \mu \mathrm{J} / \mathrm{m}^{2}$ and hence nearly negligible in all cases (Figure 2D). In general, the trend in interaction energies obtained from the calculations agreed well with the experimental results for the $\mathrm{TiO}_{2}$ and $\mathrm{SiO}_{2}$ cases, supporting that the interplay of noncovalent interfacial forces captured in the analytical model play an important role in modulating DOCP lipid vesicle-surface interactions on both surfaces. Hence, while DOCP lipid vesicle interactions with inorganic surfaces, especially $\mathrm{TiO}_{2}$, are typically analyzed in terms of coordinate bond formation, these findings establish that noncovalent interactions are a critical factor in dictating vesicle-surface adsorption behavior in this system.

\section{Effect of DOCP Fraction on $\mathrm{TiO}_{2}$ Interactions}

Guided by these observations, we proceeded to investigate how the molar fraction of DOCP lipids within DOCP/DOPC lipid vesicles affects adsorption behavior. Past studies have exclusively tested 100 mol\% DOCP lipid vesicles and we hypothesized that adjusting the DOCP lipid fraction can modulate self-assembly outcomes by virtue of balancing the interplay of noncovalent and covalent forces involved in vesicle-surface interactions. In these experiments, the buffer solution consisted of $10 \mathrm{mM}$ Tris [pH 7.5] with $150 \mathrm{mM} \mathrm{NaCl}$ in all cases. On $\mathrm{TiO}_{2}$ surfaces, the QCM-D $\Delta \mathrm{f}$ and $\Delta \mathrm{D}$ signals showed that DOCP/DOPC lipid vesicles containing 100/0, 75/25, and 50/50 molar fractions adsorbed and exhibited two-step adsorption behavior consistent with spontaneous rupture and SLB formation (Figures 3A,B). As the DOCP lipid fraction decreased from 100 to 50 mol\%, the corresponding $\Delta \mathrm{f}$ and $\Delta \mathrm{D}$ inflection points had larger magnitudes, supporting that a greater surface coverage of adsorbed vesicles and hence more vesicle-vesicle interactions were necessary to induce spontaneous rupture. ${ }^{11,36}$ This trend implies that vesicle-surface interactions became weaker at lower DOCP fractions in spite of less charge repulsion, supporting that there was less coordinate bond formation between vesicles and the surface (Figure S1 and Table S1). Strikingly, when the DOCP lipid fraction was reduced to $25 \mathrm{~mol} \%$, the lipid vesicles adsorbed monotonically but did not rupture on the $\mathrm{TiO}_{2}$ surface. Control experiments were also performed with 100 mol\% DOPC lipid vesicles (0 mol\% DOCP), which also adsorbed but did not rupture as expected. ${ }^{16}$

For lipid vesicles containing 75-100 mol\% DOCP, the corresponding final $\Delta \mathrm{f}$ and $\Delta \mathrm{D}$ shifts after buffer washing were around -24 to $-26 \mathrm{~Hz}$ and less than $1 \times 10^{-6}$, respectively, which indicate SLB formation (Figure 3C). Similar results were obtained with lipid vesicles containing $50 \mathrm{~mol} \%$ $\mathrm{DOCP}$, however, the final $\Delta \mathrm{f}$ and $\Delta \mathrm{D}$ shifts were modestly larger in that case due to incomplete rupture. ${ }^{33}$ On the other hand, for lipid vesicles containing $25 \mathrm{~mol} \% \mathrm{DOCP}$, the final $\Delta \mathrm{f}$ and $\Delta \mathrm{D}$ shifts after buffer washing were around $-100 \mathrm{~Hz}$ and $7 \times 10^{-6}$, respectively, which are consistent with intact vesicle adsorption. ${ }^{37}$ Likewise, for DOPC lipid vesicles, the final $\Delta \mathrm{f}$ and $\Delta \mathrm{D}$ shifts were around $-150 \mathrm{~Hz}$ and $16 \times 10^{-6}$, respectively, also indicating intact vesicle adsorption while the ratios of $\Delta \mathrm{f} / \Delta \mathrm{D}$ shift values support that 25/75 DOCP/DOPC lipid vesicles underwent greater adsorption-related deformation than DOPC lipid vesicles ${ }^{16}$ (Figure S2). Considering that the 25/75 DOCP/DOPC lipid vesicles are more negatively charged than DOPC lipid vesicles, the greater extent of deformation in the former case supports that covalent DOCP lipid anchoring to the $\mathrm{TiO}_{2}$ surface via coordinate bond formation enhances vesicle-surface interactions compared to 
noncovalent interactions alone. Moreover, the observed dependence on DOCP lipid fraction supports that a critical density of $\mathrm{DOCP}-\mathrm{TiO}_{2}$ anchoring sites is needed to trigger vesicle fusion/rupture and SLB formation.

(A)

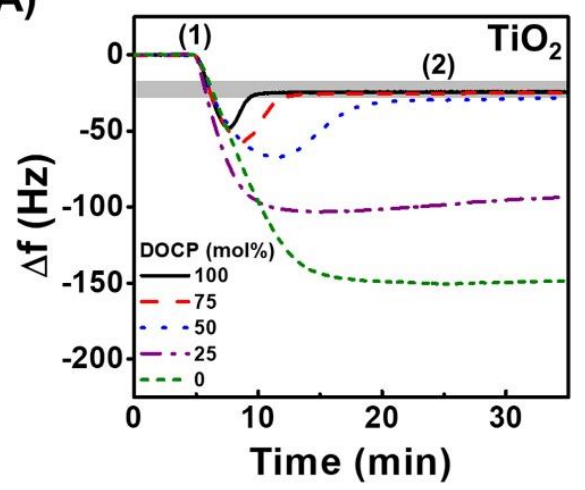

(C)

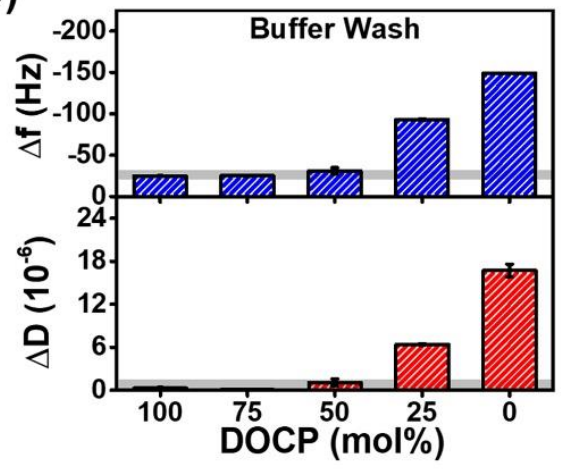

(E)

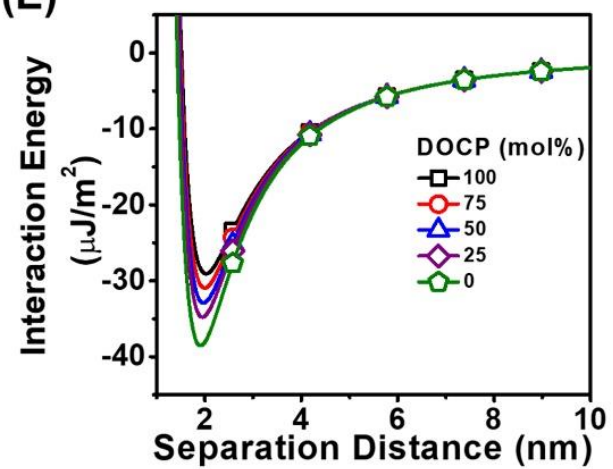

(B)

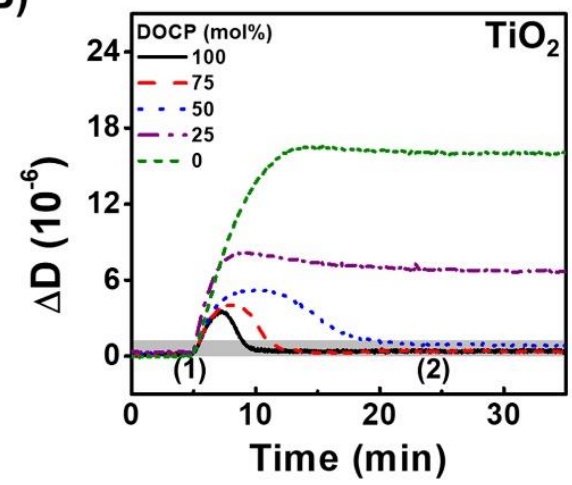

(D)
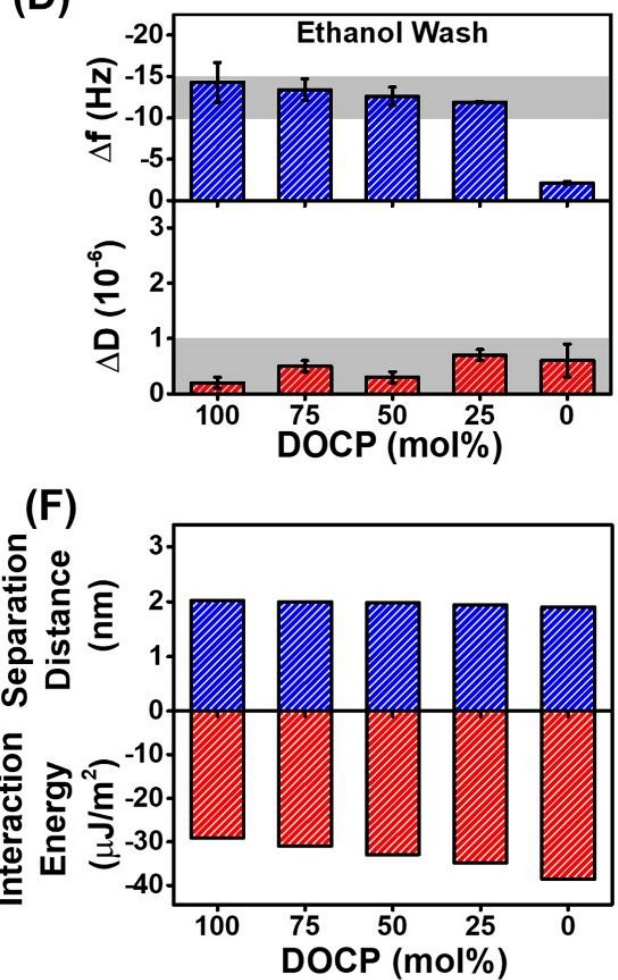

Figure 3. Tuning DOCP lipid fraction to modulate vesicle adsorption pathway on $\mathrm{TiO}_{2}$ surface. $(\mathrm{A}, \mathrm{B}) \mathrm{QCM}-\mathrm{D}$ frequency $(\Delta \mathrm{f})$ and energy dissipation $(\Delta \mathrm{D})$ signals upon DOCP/DOPC lipid vesicle addition to $\mathrm{TiO}_{2}$ surface. Labels (1) and (2) refer to vesicle addition and buffer washing, respectively. Summary of final $\Delta \mathrm{f}$ and $\Delta \mathrm{D}$ shifts after (C) buffer washing and (D) ethanol washing for adsorbed lipid layers on $\mathrm{TiO}_{2}$ surface. All reported shifts were recorded in buffer solution and are relative to the initial buffer baseline. Mean and standard deviation are reported from at least three measurements. Shaded regions in the graphs indicate typical measurement values for an SLB and lipid monolayer in panels (A-C) and (D), respectively. (E) Dependence of the total interaction energy on the vesicle-surface separation distance according to extended-DLVO model analysis. (F) Summary values of equilibrium separation distance and interaction energy. 
After lipid attachment to the $\mathrm{TiO}_{2}$ surface, an ethanol washing step was performed to remove noncovalently adsorbed lipid molecules and, after subsequently exchanging back to the buffer solution, the final $\Delta \mathrm{f}$ and $\Delta \mathrm{D}$ shifts were around -10 to $-15 \mathrm{~Hz}$ and $<1 \times 10^{-6}$, respectively, for DOCP-containing lipid vesicles relative to the initial buffer baseline (Figure 3D). This result supports that covalently attached DOCP lipid molecules remained adhered to the $\mathrm{TiO}_{2}$ surface and form a monolayer based on the surface mass density $\left(\sim 220 \mathrm{ng} / \mathrm{cm}^{2}\right)$ according to the Sauerbrey relationship. ${ }^{37}$ By contrast, the corresponding $\Delta \mathrm{f}$ and $\Delta \mathrm{D}$ shifts were only around -2 and $<1 \times 10^{-}$ 6 , respectively, in the case of DOPC lipid vesicles, which is consistent with the removal of noncovalently adsorbed DOPC lipid molecules from the $\mathrm{TiO}_{2}$ surface due to ethanol washing. Analytical calculations based on the E-DLVO model further showed that, for all tested lipid compositions, the noncovalent lipid-surface interactions were attractive (at least $-25 \mu \mathrm{J} / \mathrm{m}^{2}$ ) and tended to become more favorable with increasing DOPC lipid fraction due to a lower vesicle surface charge (Figures 3E,F). Hence, noncovalent interactions played an important role in modulating initial lipid vesicle adsorption onto the $\mathrm{TiO}_{2}$ surface while covalent interactions involving DOCP lipids were necessary to induce a sufficiently high degree of adsorption-related vesicle deformation that could trigger fusion/rupture and SLB formation. ${ }^{11}$ Importantly, a critical density of $\mathrm{DOCP}-\mathrm{TiO}_{2}$ bonding events was necessary to induce SLB formation whereas intact vesicle adsorption without rupture occurred in other cases.

\section{Effect of DOCP Fraction on $\mathrm{SiO}_{2}$ Interactions}

Similar composition-dependent experiments were performed on $\mathrm{SiO}_{2}$ surfaces and the QCM-D results showed that DOCP/DOPC lipid vesicles containing 100/0, 75/25, and 50/50 molar fractions did not adsorb onto the surface (Figures 4A,B). By contrast, DOCP/DOPC lipid vesicles containing 25 mol\% DOCP underwent monotonic adsorption while DOPC lipid vesicles exhibited two-step adsorption behavior that indicated SLB formation. For lipid vesicles containing 50-100 mol\% DOCP, the corresponding final $\Delta \mathrm{f}$ and $\Delta \mathrm{D}$ shifts after buffer washing were less than $-2 \mathrm{~Hz}$ and $0.5 \times 10^{-6}$, respectively, which are consistent with negligible adsorption (Figure 4C). For 25/75 DOCP/DOPC lipid vesicles, there was modest lipid adsorption that did not result in SLB formation, as indicated by final $\Delta \mathrm{f}$ and $\Delta \mathrm{D}$ shifts of around $-26 \mathrm{~Hz}$ and $2 \times 10^{-6}$, respectively, whereas DOPC lipid vesicles fused and ruptured to form an SLB with final $\Delta \mathrm{f}$ and $\triangle \mathrm{D}$ shifts of around $-26 \mathrm{~Hz}$ and $0.2 \times 10^{-6}$, respectively.

After ethanol washing, the $\Delta \mathrm{f}$ and $\Delta \mathrm{D}$ shifts became fully negligible in the case of lipid vesicles containing 50-100 mol\% DOCP (Figure 4D). On the other hand, for 25/75 DOCP/DOPC lipid vesicles, the final $\Delta \mathrm{f}$ and $\Delta \mathrm{D}$ shifts were around $-15 \mathrm{~Hz}$ and $2 \times 10^{-6}$, respectively, indicating that a lipid monolayer remained attached to the surface. Interestingly, this observation supports that DOCP lipids can interact with $\mathrm{SiO}_{2}$ surfaces provided that the DOCP lipid fraction is sufficiently low so that noncovalent vesicle-surface interactions - arising from reduced charge repulsion and likely involving strong hydrogen-bonding interactions between phosphate groups and silanol (SiO) groups ${ }^{38,39}$ - are favorable enough to permit initial vesicle attachment (see also another example of tight binding of phosphoinositide lipids to $\mathrm{SiO}_{2}$ surfaces ${ }^{40}$ ). Conversely, for DOPC lipid vesicles, the final $\Delta \mathrm{f}$ and $\Delta \mathrm{D}$ shifts were reduced to around $-2 \mathrm{~Hz}$ and $1 \times 10^{-6}$, respectively, which indicate that ethanol washing removed noncovalently attached DOPC lipids from the surface as expected. Furthermore, E-DLVO-based analytical calculations verified that the lipid-surface interaction energy due to noncovalent interactions was very weak for DOCP-containing lipid vesicles on $\mathrm{SiO}_{2}$ surfaces in general and was more favorable for DOPC lipid vesicles (Figures 4E,F). These 
(A)

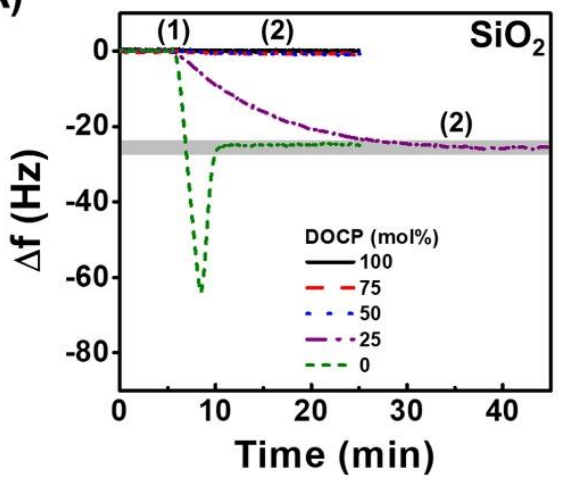

(C)

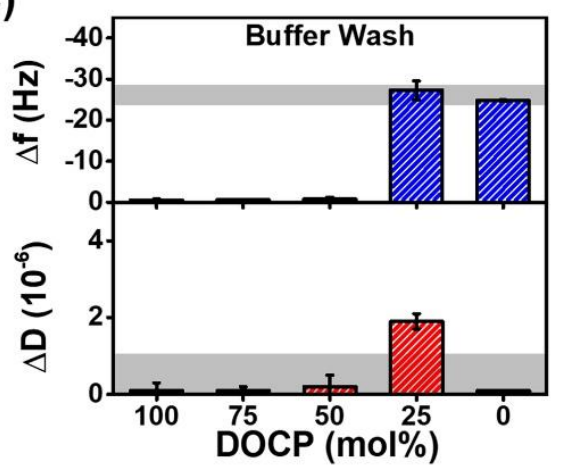

(E)

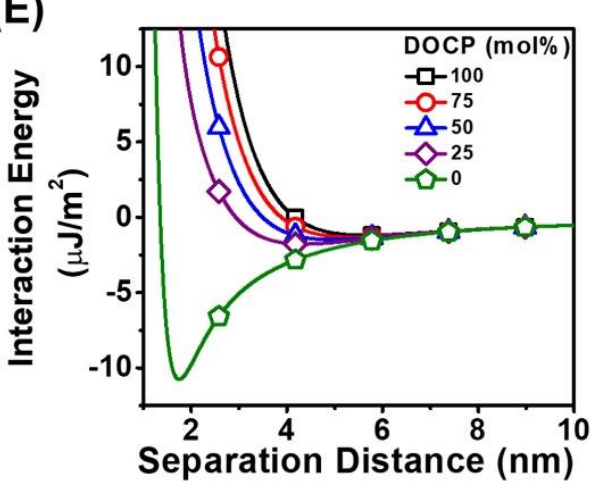

(B)

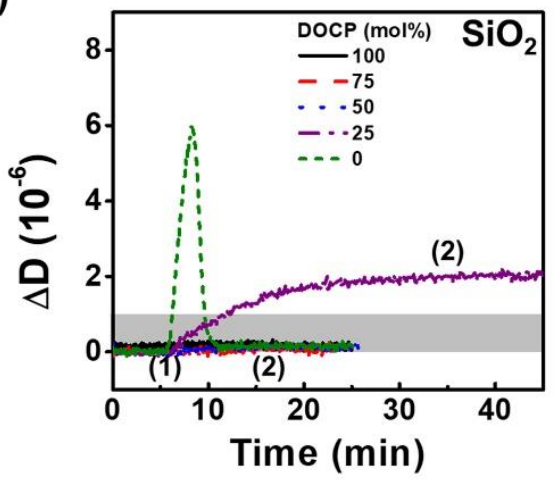

(D)

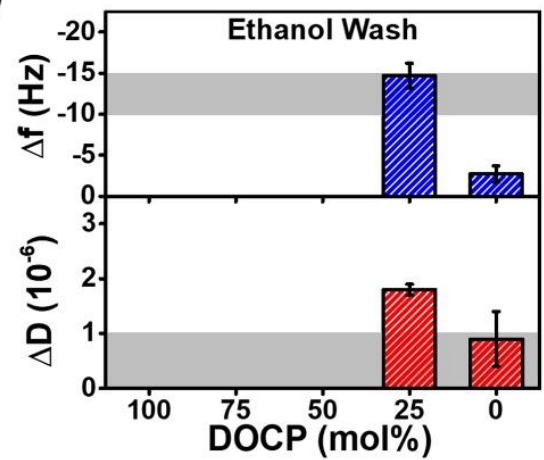

(F)

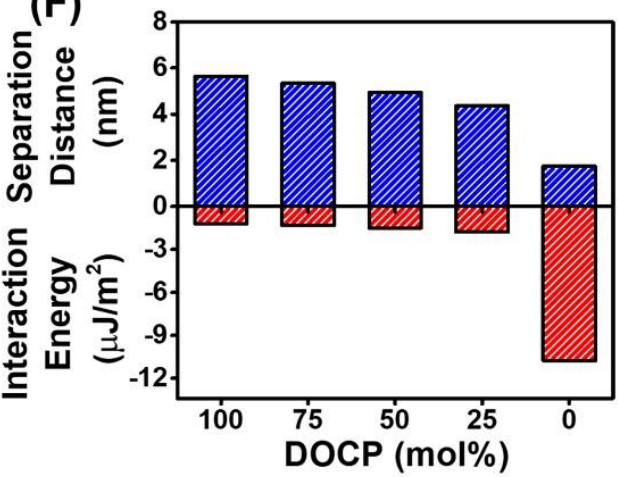

Figure 4. Tuning DOCP lipid fraction to modulate vesicle adsorption pathway on $\mathrm{SiO}_{2}$ surface. As in Figure 3, except that the substrate was $\mathrm{SiO}_{2}$.

findings are consistent with electrostatic repulsion between negatively charged DOCP-containing lipid vesicles and the $\mathrm{SiO}_{2}$ surface, which hinders rupture-induced SLB formation even in cases where moderate adsorption is possible. Together, the experimental results and analytical calculations support that DOCP lipid vesicle interactions with $\mathrm{TiO}_{2}$ and $\mathrm{SiO}_{2}$ surfaces depend on a combination of noncovalent and covalent forces in a distinct manner compared to previously studied vesicle systems where noncovalent forces alone predominate.

\section{Discussion}

It has long been recognized that vesicle adsorption behavior on inorganic surfaces such as $\mathrm{SiO}_{2}$ and $\mathrm{TiO}_{2}$ is dictated by noncovalent interfacial forces that influence the vesicle-surface interaction energy. In general, adsorbed vesicles undergo shape deformation that is influenced by an interplay 
of the membrane bending energy, deformation-related osmotic pressure energy, and vesiclesurface interaction energy. ${ }^{16,34}$ When the vesicle-surface interaction is repulsive, vesicle adsorption will not occur. On the other hand, in cases of attractive but relatively weak vesiclesurface interactions, vesicles will adsorb but only exhibit modest adsorption-related deformation and remain intact on the surface. As vesicle-surface interactions become stronger, adsorbed vesicles will undergo greater deformation that can lead to spontaneous rupture due to a combination of vesicle-surface and vesicle-vesicle interactions. On $\mathrm{TiO}_{2}$ surfaces, it is widely reported that adsorbed vesicles will remain intact while vesicles typically rupture to form an SLB on $\mathrm{SiO}_{2}$ surfaces. These tendencies are attributed to the balance of interfacial forces underpinning vesicle-surface interactions and existing efforts to modulate vesicle adsorption behavior have focused on tuning the magnitudes of these noncovalent forces.

It is thus remarkable that, in spite of a large negative surface charge that imparts significant vesiclesurface charge repulsion, DOCP lipid vesicles can form SLB coatings on $\mathrm{TiO}_{2}$ surfaces. By tracking the corresponding adsorption kinetics with the QCM-D technique, we focused on investigating the mechanistic details of how DOCP lipid vesicles form $\mathrm{SLBs}$ on $\mathrm{TiO}_{2}$ surfaces and identified how a combination of noncovalent and covalent forces is needed to drive the SLB formation process (Figure 5). First, DOCP-containing lipid vesicles must have sufficiently favorable noncovalent interactions with $\mathrm{TiO}_{2}$ surfaces to facilitate initial attachment. The attachment step is a prerequisite to facilitate bond formation between DOCP lipids and the $\mathrm{TiO}_{2}$ surfaces - enabling the reactive moieties to come into sufficiently close contact - and requires suitable solution conditions, as evidenced by the $50 \mathrm{mM} \mathrm{NaCl}$ condition in which DOCP lipid vesicles did not adsorb. Second, DOCP lipid vesicles must form a sufficient density of coordinate bonds with the $\mathrm{TiO}_{2}$ surface to induce vesicle rupture. Strikingly, the presence of even $25 \mathrm{~mol} \%$ DOCP lipids in a vesicle is insufficient to induce vesicle rupture; in that case, vesicles adsorbed and deformed to a greater extent than $100 \%$ mol DOPC lipid vesicles but did not rupture. In our experiments, at least $50 \mathrm{~mol} \%$ DOCP lipid in a vesicle was necessary to induce vesicle rupture and SLB formation.

We analogize this dependency to multivalent ligand-receptor interactions involving ligandmodified vesicles and receptor-functionalized surfaces where greater multivalency translates into more extensive shape deformation ${ }^{41}$ (see also other hybridization-related multivalent systems involving nucleic-acid-functionalized lipids ${ }^{42-44}$ ). We emphasize that DOCP-containing lipid vesicles appear to form coordinate bonds - akin to covalent anchoring - with the $\mathrm{TiO}_{2}$ surface in all applicable cases (as indicated by attached DOCP lipids after ethanol washing), but the variation in $\mathrm{DOCP}_{-} \mathrm{TiO}_{2}$ bond density per vesicle (depending on the DOCP lipid fraction in the vesicles) determines the extent of vesicle deformation and hence whether attached vesicles remain intact or rupture to form an SLB. Hence, while DOCP-TiO 2 bond formation is necessary for SLB formation on $\mathrm{TiO}_{2}$ surfaces, there are two additional key factors: (1) favorable conditions to facilitate noncovalent lipid vesicle adsorption onto the $\mathrm{TiO}_{2}$ surface, otherwise, coordinate bond formation cannot occur because lipid headgroups and the $\mathrm{TiO}_{2}$ surface cannot come into sufficiently close proximity; and (2) a minimum density of $\mathrm{DOCP}-\mathrm{TiO}_{2}$ bonds, which function as covalent anchoring points, in order to drive extensive vesicle deformation that can lead to fusion/rupture triggering SLB formation. While conventional vesicle fusion strategies involve enhancing noncovalent interactions to promote greater vesicle deformation, a unique aspect of the present system is that a combination of noncovalent and covalent forces is needed to drive DOCP-containing lipid vesicle fusion on $\mathrm{TiO}_{2}$ surfaces. 


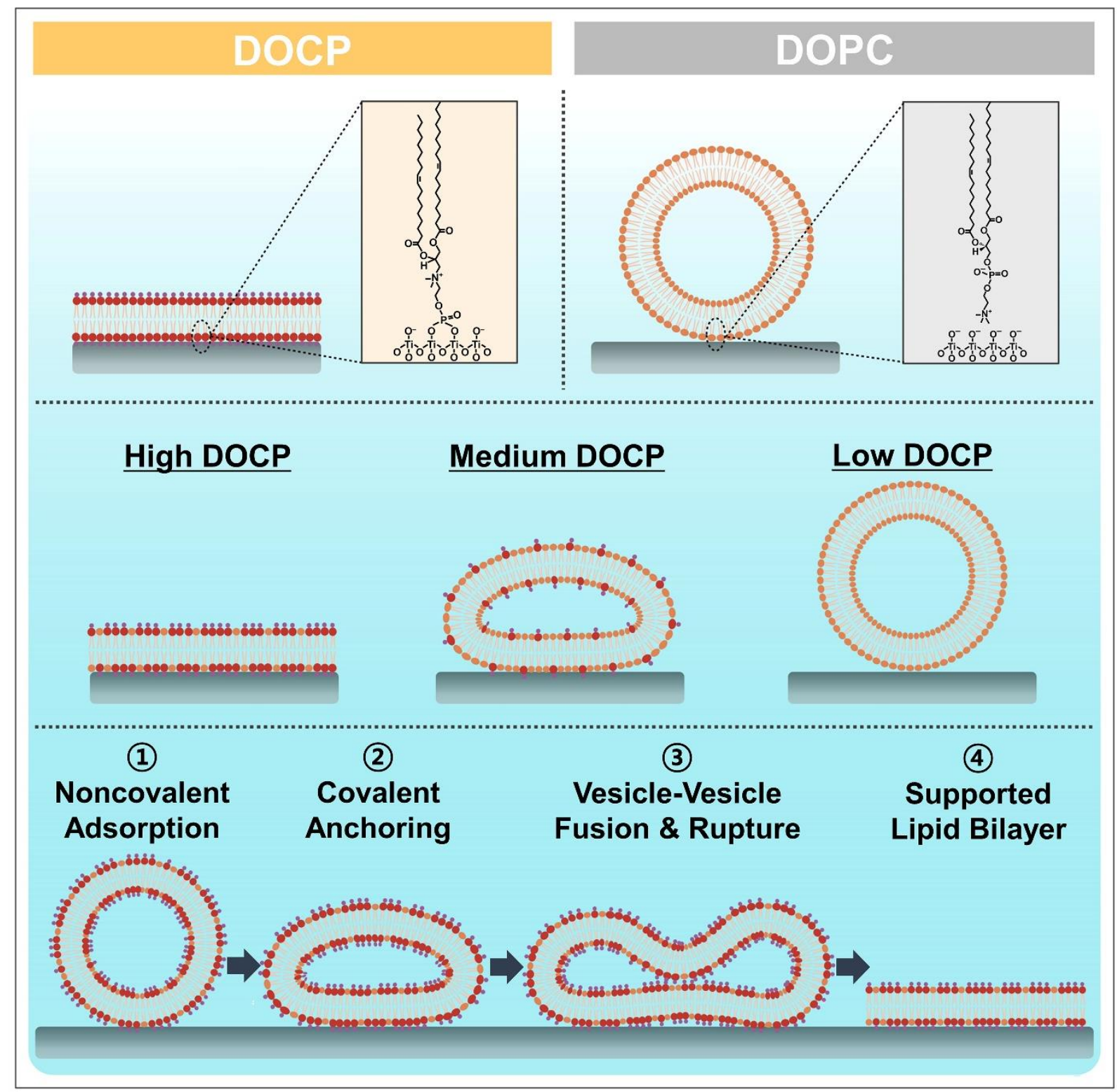

Figure 5. Schematic summary of how noncovalent and covalent forces jointly contribute to DOCP lipid vesicle interactions with $\mathrm{TiO}_{2}$ surfaces. Top: DOCP and DOPC lipid molecules mainly interact with $\mathrm{TiO}_{2}$ surfaces though coordinate bond formation and noncovalent adhesion, respectively. Middle: The interaction of DOCP-containing lipid vesicles with $\mathrm{TiO}_{2}$ surfaces is affected by the DOCP fraction in the vesicles. At high DOCP fractions, supported lipid bilayer formation can occur due to strong vesicle-surface interactions while the interactions become progressively weaker at lower DOCP fractions. At intermediate DOCP fractions, vesicles adsorb and undergo deformation due to moderately strong interactions but do not rupture. At low DOCP fractions, vesicles adsorb but do not undergo extensive deformation due to relatively weak interactions. Bottom: Mechanism of how DOCP lipid vesicles interact with $\mathrm{TiO}_{2}$ surface to form a supported lipid bilayer coating, which involves (1) noncovalent adsorption, (2) covalent anchoring due to $\mathrm{DOCP}-\mathrm{TiO}_{2}$ coordinate bond formation whereby greater bond density drives more extensive vesicle deformation, (3) fusion of deformed vesicles leading to rupture, and (4) lipid re-assembly to form a supported lipid bilayer. 
Another striking feature is that, even for $100 \mathrm{~mol} \%$ DOCP lipid vesicles where the highest possible density of covalent anchoring points can form, the two-step adsorption kinetics observed in the QCM-D data indicate that vesicle-surface interactions are insufficient to cause rupture of individually attached vesicles and instead a combination of vesicle-surface and vesicle-vesicle interactions is needed to trigger the vesicle fusion and rupture process. This finding further highlights the distinct chemical features of DOCP lipid vesicle interactions on $\mathrm{TiO}_{2}$ surfaces compared to more conventionally studied PC lipid vesicle interactions on $\mathrm{SiO}_{2}$ surfaces where vesicle-surface interactions alone can induce rupture of individually attached vesicles, as dictated by noncovalent interfacial forces alone (especially electrostatic forces ${ }^{45}$ ).

In marked contrast, DOCP-containing lipid vesicles are mainly repelled from $\mathrm{SiO}_{2}$ surfaces due to extensive charge repulsion and, even in the limited cases where modest adsorption occurs, the extent of vesicle deformation is still insufficient to promote vesicle fusion/rupture. These observations highlight the importance of both noncovalent and covalent forces in utilizing DOCP lipid vesicles to form SLB coatings on inorganic surfaces.

\section{Conclusion}

In this work, we have demonstrated how balancing the interplay of noncovalent and covalent forces can tune the self-assembly pathway of DOCP lipid vesicles interacting with $\mathrm{TiO}_{2}$ surfaces. Compared to other systems involving lipid vesicle interactions with inorganic surfaces, this system is unique in several respects. First, the noncovalent interactions between lipid vesicles and $\mathrm{TiO}_{2}$ surfaces are relatively weak overall (likely due to steric-hydration repulsion ${ }^{16,24}$ ) and $\mathrm{DOCP}^{-\mathrm{TiO}_{2}}$ coordinate bond formation enables covalent anchoring, which effectively serves as a potentiating force to enhance vesicle-surface interactions. Second, a minimum density of $\mathrm{DOCP}-\mathrm{TiO}_{2}$ coordinate bonds - inferred from the DOCP fraction in vesicles - is needed to induce sufficiently high levels of vesicle deformation that can lead to fusion and rupture. At lower densities, covalent anchoring can still induce modest deformation of attached vesicles, but they do not fuse and rupture. Third, even at the highest DOCP fraction, vesicle-surface interactions by themselves are insufficient to drive rupture of individually attached vesicles on $\mathrm{TiO}_{2}$ surfaces and instead a combination of vesicle-surface and vesicle-vesicle interactions is needed.

The latter observation demonstrates that the combination of noncovalent and covalent forces that drives DOCP lipid vesicle adsorption and rupture on $\mathrm{TiO}_{2}$ surfaces is still relatively weak considering that noncovalent forces alone can induce sufficiently strong vesicle-surface interactions to rupture individually attached PC lipid vesicles on $\mathrm{SiO}_{2}$ surfaces in some cases. As such, the interfacial chemistry underpinning DOCP lipid vesicle interactions with $\mathrm{TiO}_{2}$ surfaces involves a subtle interplay of noncovalent and covalent forces and can induce SLB formation in select conditions. These findings also help to understand the interaction behavior of DOCP lipid vesicles with $\mathrm{SiO}_{2}$ surfaces and more broadly highlight opportunities to modulate lipid selfassembly at inorganic surfaces by harnessing noncovalent and covalent forces in tandem, especially in cases involving coordination chemistry. 


\section{Associated Content}

\section{Supporting Information}

Additional experimental details and results, including materials and methods, vesicle size and zeta potential characterization, and analysis of vesicle deformation as well as details of interfacial force modeling calculations.

\section{$\underline{\text { Author Information }}$}

\section{Corresponding Author}

*njcho@ntu.edu.sg

*jjackman@skku.edu

\section{Author Contributions}

All authors have given approval to the final version of the manuscript.

\section{Notes}

The authors declare no competing financial interest.

\section{Acknowledgment}

This work was supported by the National Research Foundation of Singapore through a Proof-ofConcept grant (NRF2015NRF-POC0001-19), by the Ministry of Education (MOE) in Singapore under Grant AcRF TIER1-2020-T1-002-032 (RG111/20), and by the National Research Foundation of Korea (NRF) grants funded by the Korean government (MSIT) (Nos. 2020R1C1C1004385 and 2021R1A4A1032782). In addition, this work was supported by the International Research \& Development Program of the National Research Foundation of Korea (NRF) funded by the Ministry of Science and ICT (2020K1A3A1A39112724). Graphics were created with BioRender.com under an academic lab subscription.

\section{Abbreviations}

DOCP, 2-((2,3-bis(oleoyloxy)propyl)dimethylammonio)ethyl hydrogen phosphate; DOPC, 1,2dioleoyl-sn-glycero-3-phosphocholine; E-DLVO, extended Derjaguin-Landau-Verwey-Overbeek; QCM-D, quartz crystal microbalance-dissipation PC, phosphatidylcholine; $\mathrm{SiO}_{2}$, silicon dioxide; $\mathrm{TiO}_{2}$, titanium dioxide; SLB, supported lipid bilayer.

\section{$\underline{\text { References }}$}

(1) Castellana, E. T.; Cremer, P. S. Solid supported lipid bilayers: from biophysical studies to sensor design. Surf. Sci. Rep. 2006, 61 (10), 429-444.

(2) Tero, R. Substrate effects on the formation process, structure and physicochemical properties of supported lipid bilayers. Materials 2012, 5 (12), 2658-2680.

(3) Liu, J. Interfacing zwitterionic liposomes with inorganic nanomaterials: surface forces, membrane integrity, and applications. Langmuir 2016, 32 (18), 4393-4404.

(4) Jackman, J. A.; Cho, N.-J. Supported lipid bilayer formation: beyond vesicle fusion. Langmuir 2020, 36 (6), 1387-1400. 
(5) Sut, T. N.; Yoon, B. K.; Jeon, W.-Y.; Jackman, J. A.; Cho, N.-J. Supported lipid bilayer coatings: fabrication, bioconjugation, and diagnostic applications. Appl. Mater. Today 2021, 25, 101183.

(6) Ramanathan, M.; Shrestha, L. K.; Mori, T.; Ji, Q.; Hill, J. P.; Ariga, K. Amphiphile nanoarchitectonics: from basic physical chemistry to advanced applications. Phys. Chem. Chem. Phys. 2013, 15 (26), 10580-10611.

(7) Ariga, K.; Watanabe, S.; Mori, T.; Takeya, J. Soft 2D nanoarchitectonics. NPG Asia Mater. 2018, 10 (4), 90-106.

(8) Keller, C.; Glasmästar, K.; Zhdanov, V.; Kasemo, B. Formation of supported membranes from vesicles. Phys. Rev. Lett. 2000, 84 (23), 5443.

(9) Johnson, J. M.; Ha, T.; Chu, S.; Boxer, S. G. Early steps of supported bilayer formation probed by single vesicle fluorescence assays. Biophys. J. 2002, 83 (6), 3371-3379.

(10) Schönherr, H.; Johnson, J. M.; Lenz, P.; Frank, C. W.; Boxer, S. G. Vesicle adsorption and lipid bilayer formation on glass studied by atomic force microscopy. Langmuir 2004, 20 (26), 11600-11606.

(11) Richter, R. P.; Bérat, R.; Brisson, A. R. Formation of solid-supported lipid bilayers: an integrated view. Langmuir 2006, 22 (8), 3497-3505.

(12) Mapar, M.; Jõemetsa, S.; Pace, H.; Zhdanov, V. P.; Agnarsson, B.; Höök, F. Spatiotemporal kinetics of supported lipid bilayer formation on glass via vesicle adsorption and rupture. J. Phys. Chem. Lett. 2018, 9 (17), 5143-5149.

(13) Anderson, T. H.; Min, Y.; Weirich, K. L.; Zeng, H.; Fygenson, D.; Israelachvili, J. N. Formation of supported bilayers on silica substrates. Langmuir 2009, 25 (12), 6997-7005.

(14) Lind, T. K.; Cárdenas, M. Understanding the formation of supported lipid bilayers via vesicle fusion - a case that exemplifies the need for the complementary method approach. Biointerphases 2016, 11 (2), 020801.

(15) Schneemilch, M.; Quirke, N. Free energy of adsorption of supported lipid bilayers from molecular dynamics simulation. Chem. Phys. Lett. 2016, 664, 199-204.

(16) Reimhult, E.; Höök, F.; Kasemo, B. Intact vesicle adsorption and supported biomembrane formation from vesicles in solution: influence of surface chemistry, vesicle size, temperature, and osmotic pressure. Langmuir 2003, 19 (5), 1681-1691.

(17) Cremer, P. S.; Boxer, S. G. Formation and spreading of lipid bilayers on planar glass supports. J. Phys. Chem. B 1999, 103 (13), 2554-2559.

(18) Schneemilch, M.; Quirke, N. Free energy of adhesion of lipid bilayers on silica surfaces. $J$. Chem. Phys. 2018, 148 (19), 194704.

(19) Schneemilch, M.; Quirke, N. Free energy of adhesion of lipid bilayers on titania surfaces. $J$. Chem. Phys. 2019, 151 (13), 134707. 
(20) Biswas, K. H.; Jackman, J. A.; Park, J. H.; Groves, J. T.; Cho, N.-J. Interfacial forces dictate the pathway of phospholipid vesicle adsorption onto silicon dioxide surfaces. Langmuir 2018, 34 (4), 1775-1782.

(21) Reimhult, E.; Höök, F.; Kasemo, B. Vesicle adsorption on $\mathrm{SiO}_{2}$ and $\mathrm{TiO}_{2}$ : dependence on vesicle size. J. Chem. Phys. 2002, 117 (16), 7401-7404.

(22) Jackman, J. A.; Špačková, B.; Linardy, E.; Kim, M. C.; Yoon, B. K.; Homola, J.; Cho, N.-J. Nanoplasmonic ruler to measure lipid vesicle deformation. Chem. Commun. 2016, 52, 76-79.

(23) Ferhan, A. R.; Spackova, B.; Jackman, J. A.; Ma, G. J.; Sut, T. N.; Homola, J.; Cho, N.-J. Nanoplasmonic ruler for measuring separation distance between supported lipid bilayers and oxide surfaces. Anal. Chem. 2018, 90 (21), 12503-12511.

(24) Jackman, J. A.; Zan, G. H.; Zhao, Z.; Cho, N.-J. Contribution of the hydration force to vesicle adhesion on titanium oxide. Langmuir 2014, 30 (19), 5368-5372.

(25) Cho, N.-J.; Jackman, J. A.; Liu, M.; Frank, C. W. pH-Driven assembly of various supported lipid platforms: a comparative study on silicon oxide and titanium oxide. Langmuir 2011, 27 (7), 3739-3748.

(26) Ferhan, A. R.; Yoon, B. K.; Park, S.; Sut, T. N.; Chin, H.; Park, J. H.; Jackman, J. A.; Cho, N.-J. Solvent-assisted preparation of supported lipid bilayers. Nat. Protoc. 2019, 14 (7), 20912118.

(27) Tabaei, S. R.; Jackman, J. A.; Kim, S.-O.; Zhdanov, V. P.; Cho, N.-J. Solvent-assisted lipid self-assembly at hydrophilic surfaces: factors influencing the formation of supported membranes. Langmuir 2015, 31 (10), 3125-3134.

(28) Liu, Y.; Wang, F.; Liu, J. Headgroup-inversed liposomes: biointerfaces, supported bilayers and applications. Langmuir 2018, 34 (32), 9337-9348.

(29) Perttu, E. K.; Kohli, A. G.; Szoka Jr, F. C. Inverse-phosphocholine lipids: a remix of a common phospholipid. J. Am. Chem. Soc. 2012, 134 (10), 4485-4488.

(30) Wang, F.; Liu, J. A stable lipid/TiO 2 interface with headgroup-inversed phosphocholine and a comparison with $\mathrm{SiO}_{2}$. J. Am. Chem. Soc. 2015, 137 (36), 11736-11742.

(31) Wang, F.; Zhang, X.; Liu, Y.; Lin, Z. Y.; Liu, B.; Liu, J. Profiling metal oxides with lipids: magnetic liposomal nanoparticles displaying DNA and proteins. Angew. Chem., Int. Ed. 2016, 55 (39), 12063-12067.

(32) Wang, X.; Li, X.; Wang, H.; Zhang, X.; Zhang, L.; Wang, F.; Liu, J. Charge and coordination directed liposome fusion onto $\mathrm{SiO}_{2}$ and $\mathrm{TiO}_{2}$ nanoparticles. Langmuir 2018, 35 (5), 1672-1681.

(33) Cho, N.-J.; Frank, C. W.; Kasemo, B.; Höök, F. Quartz crystal microbalance with dissipation monitoring of supported lipid bilayers on various substrates. Nat. Protoc. 2010, 5 (6), 1096-1106.

(34) Jackman, J. A.; Choi, J.-H.; Zhdanov, V. P.; Cho, N.-J. Influence of osmotic pressure on adhesion of lipid vesicles to solid supports. Langmuir 2013, 29 (36), 11375-11384. 
(35) Jackman, J. A.; Tabaei, S. R.; Zhao, Z.; Yorulmaz, S.; Cho, N.-J. Self-assembly formation of lipid bilayer coatings on bare aluminum oxide: overcoming the force of interfacial water. $A C S$ Appl. Mater. Interfaces 2015, 7 (1), 959-968.

(36) Seantier, B.; Kasemo, B. Influence of mono- and divalent ions on the formation of supported phospholipid bilayers via vesicle adsorption. Langmuir 2009, 25 (10), 5767-5772.

(37) Keller, C. A.; Kasemo, B. Surface specific kinetics of lipid vesicle adsorption measured with a quartz crystal microbalance. Biophys. J. 1998, 75 (3), 1397-1402.

(38) Murashov, V. V.; Leszczynski, J. Adsorption of the phosphate groups on silica hydroxyls: an ab initio study. J. Phys. Chem. A 1999, 103 (9), 1228-1238.

(39) Cai, L.; Sun, Q.; Bao, M.; Ma, H.; Yuan, C.; Xu, W. Competition between hydrogen bonds and coordination bonds steered by the surface molecular coverage. ACS Nano 2017, 11 (4), 37273732 .

(40) Sun, S.; Liu, C.; Rodriguez Melendez, D.; Yang, T.; Cremer, P. S. Immobilization of phosphatidylinositides revealed by bilayer leaflet decoupling. J. Am. Chem. Soc. 2020, 142 (30), 13003-13010.

(41) Park, H.; Sut, T. N.; Yoon, B. K.; Zhdanov, V. P.; Cho, N.-J.; Jackman, J. A. Unraveling how multivalency triggers shape deformation of sub-100 nm lipid vesicles. J. Phys. Chem. Lett. 2021, $12(28), 6722-6729$.

(42) Chung, M.; Lowe, R. D.; Chan, Y.-H. M.; Ganesan, P. V.; Boxer, S. G. DNA-tethered membranes formed by giant vesicle rupture. J. Struct. Biol. 2009, 168 (1), 190-199.

(43) Chung, M.; Boxer, S. G. Stability of DNA-tethered lipid membranes with mobile tethers. Langmuir 2011, 27 (9), 5492-5497.

(44) Chung, M.; Koo, B. J.; Boxer, S. G. Formation and analysis of topographical domains between lipid membranes tethered by DNA hybrids of different lengths. Faraday Discuss. 2013, 161, 333345.

(45) Jackman, J. A.; Cho, N.-J.; Duran, R. S.; Frank, C. W. Interfacial binding dynamics of bee venom phospholipase $\mathrm{A}_{2}$ investigated by dynamic light scattering and quartz crystal microbalance. Langmuir 2010, 26 (6), 4103-4112. 